\title{
CT-guided localization techniques of small pulmonary nodules: a prospective non-randomized controlled study on pulmonary nodule localization needle and methylene blue staining with surgical glue
}

\author{
Jian Kong ${ }^{1 \#}$, Jianxi Guo ${ }^{1 \#}$, Hua Zhang ${ }^{1}$, Yong Li $^{1}$, Guangsuo Wang ${ }^{2}$, Yanfang Zhang ${ }^{1}$ \\ ${ }^{1}$ Department of Interventional Radiology, Shenzhen People's Hospital (The Second Clinical Medical College, Jinan University; The First Affiliated \\ Hospital, Southern University of Science and Technology), Shenzhen, China; ${ }^{2}$ Department of Thoracic Surgery, Shenzhen People's Hospital (The \\ Second Clinical Medical College, Jinan University, The First Affiliated Hospital, Southern University of Science and Technology), Shenzhen, China \\ Contributions: (I) Conception and design: J Kong, J Guo, G Wang, Y Zhang; (II) Administrative support: G Wang, Y Zhang ; (III) Provision of study \\ materials or patients: J Kong, J Guo, H Zhang, Y Li, G Wang, Y Zhang ; (IV) Collection and assembly of data: J Kong, J Guo, H Zhang, Y Li ; (V) \\ Data analysis and interpretation: J Kong, J Guo, H Zhang; (VI) Manuscript writing: All authors; (VII) Final approval of manuscript: All authors. \\ \#These authors contributed equally to this work. \\ Correspondence to: Yanfang Zhang. Department of Interventional Radiology, Shenzhen People's Hospital (The Second Clinical Medical College, Jinan \\ University; The First Affiliated Hospital, Southern University of Science and Technology), Shenzhen 518020, China. Email: 1015430122@qq.com; \\ Guangsuo Wang. Department of Thoracic Surgery, Shenzhen People's Hospital (The Second Clinical Medical College, Jinan University; The First \\ Affiliated Hospital, Southern University of Science and Technology), Shenzhen 518020, China. Email: 908611104@qq.com.
}

Background: Thoracoscopic resection of small pulmonary nodules (SPNs) is challenging. Accurate preoperative computed tomography-guided localization of SPNs is key to successful rection. The aim of the present study was to evaluate the clinical value of a novel localization needle and methylene blue staining combined with surgical glue (MBSG) and to explore the risk factors for post-localization complications.

Methods: This prospective, non-randomized controlled study was conducted on 110 patients who received either MBSG or novel needle localization prior to video-assisted thoracoscopic surgery (VATS) from January 2019 to December 2019 at Shenzhen People's Hospital. The primary endpoints were the safety and the success rates of the 2 localization techniques. The secondary endpoints were operative time and feasibility.

Results: The 110 patients were categorized into 2 groups: the MBSG group $(n=84)$ and the pulmonary nodule localization needle group $(n=26)$. The success rate of pre-VATS localization was $100 \%$ in both groups. No deaths or serious complications occurred during localization. The rates of pneumothorax, pulmonary hemorrhage, and localization-induced cough were $38.1 \%, 25 \%$, and $7.14 \%$, respectively, in the MBSG group, and 26.92\%, 19.23\%, and 0\%, respectively, in the pulmonary nodule localization needle group. Differences between the 2 groups were not statistically significant $(\mathrm{P}>0.05)$. Total complication rate and the incidence of pain were significantly lower in the pulmonary nodule localization needle group $\left(\chi^{2}=4.441\right.$ and 4.295, respectively; $\mathrm{P}<0.05)$. The difference in operative time between the 2 groups was not statistically significant $(\mathrm{P}>0.05)$. Dye diffusion occurred in 2 patients in the MBSG group; however, it had no impact on VATS or on the pathological analysis. Neither displacement nor dislocation was observed in the pulmonary nodule localization needle group. Logistic regression analysis showed that the localization technique was an independent risk factor for total complications (odds ratio: 2.634, 95\% confidence interval: 1.022-6.789, $\mathrm{P}<0.05)$.

Conclusions: Both techniques can localize SPNs effectively prior to VATS. The pulmonary nodule localization needle technique has a lower incidence of complications.

Keywords: Pulmonary nodule localization; computed tomography-guided localization; pulmonary nodule localization needle; video-assisted thoracoscopic surgery (VATS) 
Submitted Sep 17, 2020. Accepted for publication Nov 13, 2020.

doi: $10.21037 /$ jtd-20-3147

View this article at: http://dx.doi.org/10.21037/jtd-20-3147

\section{Introduction}

With the recent increase in regular physical examinations and the widespread use of low-dose chest computed tomography (CT), the detection rate of small pulmonary nodules (SPNs) has increased significantly. However, the diagnosis and treatment of SPNs create new challenges for clinicians. It is difficult to differentiate benign from malignant SPNs based on CT scans. Histopathological tests are required for the accurate diagnosis of SPNs that are suspected to be malignant (1). The National Comprehensive Cancer Network guidelines for lung cancer screening (version 1.2020) recommend that needle biopsy or surgical excision be performed to determine the nature of SPNs when they show a tendency for malignant degeneration so that follow-up and treatment plans can be initiated (2). It can be difficult to determine the pathological results of SPNs with transtracheal or percutaneous biopsy $(3,4)$. In such cases, surgical excision is required for more detailed pathological results.

Video-assisted thoracoscopic surgery (VATS) is a minimally invasive procedure, and has the advantages of less surgical trauma, faster postoperative recovery, and fewer complications (5-7). However, VATS usually cannot accurately localize ground-glass or deep pleural nodules, resulting in thoracotomy or extended excision (8). Therefore, various pre-VATS localization methods have been proposed. Currently, in clinical settings, the two most commonly used methods are CT-guided hookwire localization and CT-guided methylene blue staining combined with surgical glue (MBSG); however, both these techniques have limitations.

A novel SPN localization needle modified based on hookwire has recently been used in the clinical setting (9). Our research team found that the pulmonary nodule localization needle not only reduces the rates of hookwire detachment and complications, such as localized pain, but also avoids dye diffusion, which occurs when MBSG is used. However, no control study has been done to compare MBSG with pulmonary nodule localization needle. The aim of the present study was to analyze the safety and effectiveness of both techniques, and to explore the risk factors related to post-localization complications.

We present the following article in accordance with the
TREND reporting checklist (available at http://dx.doi. org/10.21037/jtd-20-3147).

\section{Methods}

This present study was reviewed and approved by the Ethics Committee of Shenzhen People's Hospital, China (no. LLKY-2020325). Signed informed consent was provided by patients and/or immediate family members preoperatively. All procedures performed in this study involving human participants were in accordance with the Declaration of Helsinki (as revised in 2013).

The present study was a single-center, prospective, nonrandomized controlled study. The inclusion criteria were as follows (9): (I) age between 18 and 80 years; (II) small lung nodules with a maximum diameter of $>5 \mathrm{~mm}$, but $\leq 15 \mathrm{~mm}$, and diagnosed as malignant by a multidisciplinary team of radiologists and thoracic surgeons; (III) no imaging signs of end-stage tumors; (IV) no obvious emphysema, pulmonary hypertension, or pulmonary fibrosis; (V) no obvious large vessels, pulmonary bullae, or other important tissues or structures in the planned puncture route; (VII) normal coagulation; and (VIII) intraoperative and postoperative pathological results. The exclusion criteria were as follows: (I) medical conditions that were not suitable for pre-VATS localization, such as severe pleural effusion or lung injury; (II) severe heart/liver/lung/brain disease or severe infections 1 month prior to localization; and (III) distant metastasis. All of the included patients only underwent pre-VATS localization once.

\section{Localization techniques}

Prior to localization, imaging data were reviewed by thoracic surgeons. The puncture route was then determined according to the planned VATS approach. Attention was paid to avoid blood vessels, trachea, and interlobular septa. A 20-slice helical CT scanner (Siemens Healthcare, Erlange, Germany) was used to guide puncturing. The puncture site was re-confirmed and the puncture route was planned according to chest CT imaging. Two different localization techniques were used, and the patients were assigned to 1 of the 2 groups according to the localization 

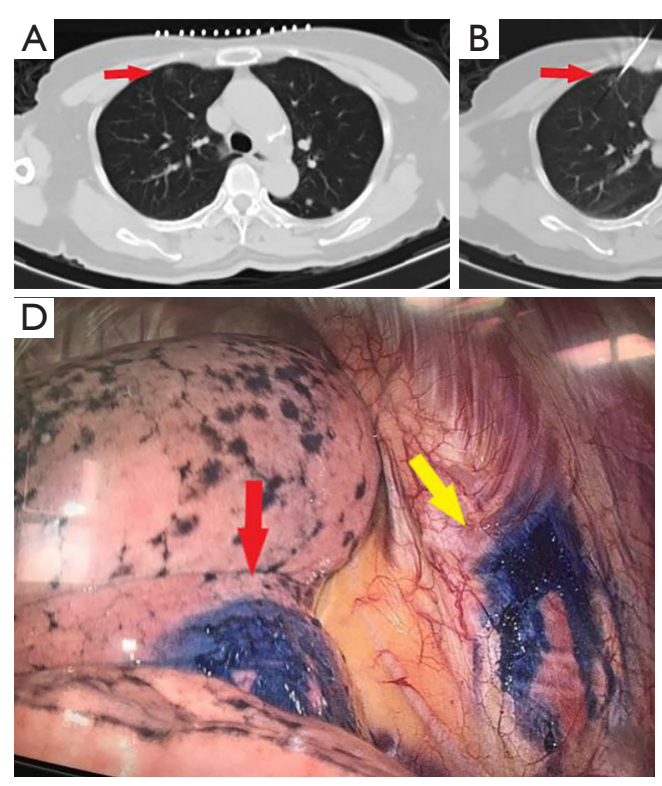
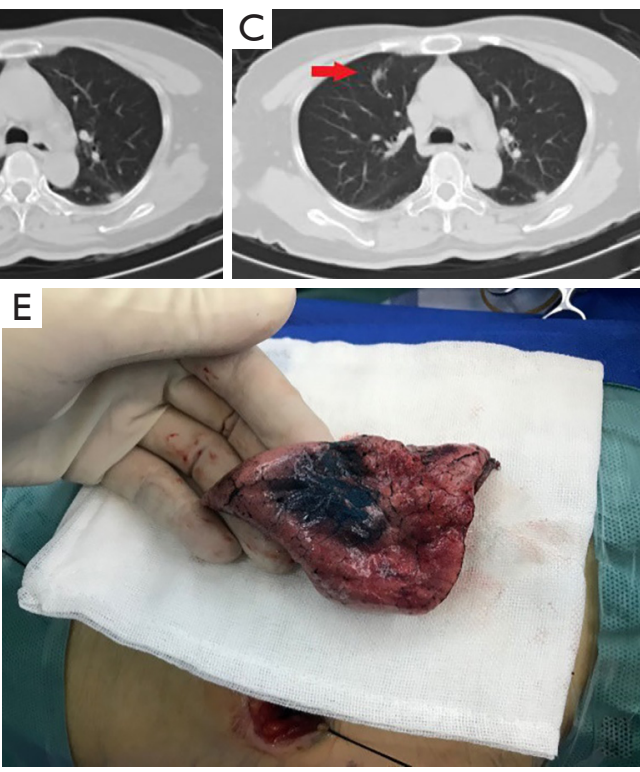

Figure 1 Computed tomography (CT)-guided methylene blue staining combined with surgical glue on a 65 -year-old female with invasive adenocarcinoma in the right upper lung lobe. (A) CT image showing a small pulmonary nodule (arrow) in the right upper lung lobe; (B) CT image showing the coaxial needle placement 5-10 mm caudal to the nodule (arrow); (C) CT image showing the change after the injection of the suspension of surgical glue and methylene blue (arrow); (D) methylene blue staining of the thoracic wall (yellow arrow) and pleura (red arrow) during video-assisted thoracoscopic surgery; (E) wedge resection is performed in the right upper lung lobe.

technique.

In the MBSG group (5 mL of surgical glue, Guangzhou Baiyun Surgical Glue, Guangzhou, China; $2 \mathrm{~mL}$ of methylene blue, Jumpcan Pharmaceutical Group, Taixing, China), a 17-gauge coaxial trocar (Bard, Salt Lake City, UT, USA) was used. The tip was placed $5-10 \mathrm{~mm}$ from the boundary of the target nodule. The stylet was removed after CT confirmation that the tip was in an appropriate position. Approximately $0.2-0.5 \mathrm{~mL}$ of the suspension (mixture of $2 \mathrm{~mL}$ methylene blue and $1 \mathrm{~mL}$ surgical glue) was injected using a $3 \mathrm{ml}$ syringe with a screw-on tip via the trocar. The needle tip was pulled back; when the tip was very close to the pleura, the trocar was removed and the puncture site was dressed (Figure 1).

In the pulmonary nodule localization needle group (Ningbo Senscure Biotechnology, Ningbo, China), the needle tip was placed directly $5-10 \mathrm{~mm}$ from the boundary of the target nodule via the puncture route. The release buckle was removed, and the pusher was pushed to the marked line. The anchor claw was released and fixed to the nodule or its boundary. The pusher was then removed before the tip was pulled back to a position between the outer wall of the thoracic cavity and the lungs. The pusher was then reinserted into the needle, pushing the tri-color suture completely out of the needle. Both the needle and the pusher were then removed (Figure 2).

Another chest CT scan was performed after localization. The localization effect and the spatial relation between the lesion and the location marker were reported to the surgeons.

\section{VATS and pathological examination}

The patients were transferred to the operating room within $12 \mathrm{~h}$ following localization. They were placed under general anesthesia and underwent 1-lung ventilation. An incision of about $3 \mathrm{~cm}$ was made in the $4 \mathrm{th} / 5$ th intercostal space at the anterior axillary line. A pair of oval forceps and/or fingers were used for inspection. Another incision was cut in the $8^{\text {th }}$ intercostal space at the midaxillary line on the side of the unhealthy lung to insert the thoracoscope.

In the MBSG group, nodules were located by the naked eye or via instrument palpation. A wedge or segmental resection of the corresponding lung tissues was then performed according to the spatial relation between the nodules and the location marker revealed by the prelocalization and post-localization CT images.

In the pulmonary nodule localization needle group, 

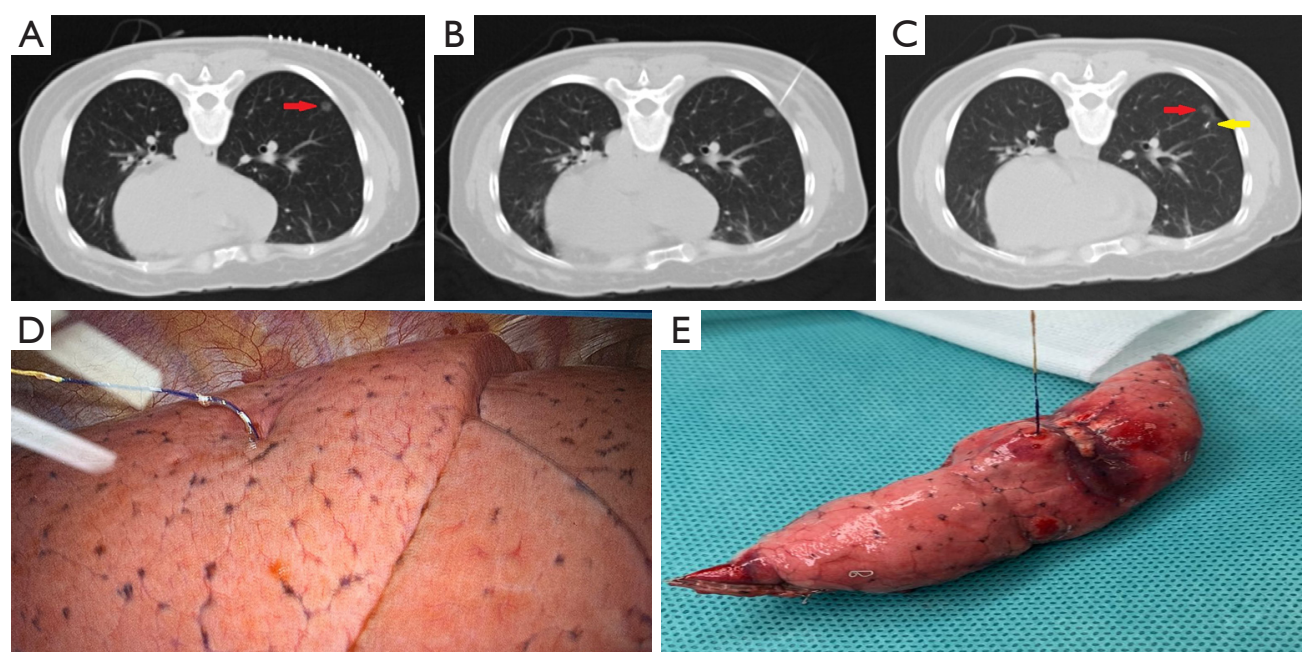

Figure 2 Computed tomography (CT)-guided pulmonary nodule localization needle on a 40-year-old female with minimally invasive adenocarcinoma in the right middle lung lobe. (A) CT image showing a small pulmonary nodule (arrow) in the right lower lobe. (B) CT image showing the coaxial needle placement 5-10 mm caudal to the nodule. (C) CT image showing the spatial relation between the anchor claw (yellow arrow) and the nodule (red arrow). A small associated pneumothorax and perilesional hemorrhage is noted. (D) Part of the tri-colored suture is seen outside the lung during video-assisted thoracoscopic surgery. (E) Wedge resection of the right lower lobe is successfully performed.

the suture was pulled gently, and the lesion was localized according to the reading on the scale. Wedge resection was then performed. Segmental resection was performed on deep nodules or on nodules for which wedge resection was not suitable.

Pathological examination of frozen tissue sections was done quickly. Surgery was ceased when there was a benign pathological result. Simple wedge or segmental resection was performed when the result revealed a precancerous lesion or minimally invasive adenocarcinoma. Lobectomy combined with systemic lymph node dissection was performed on patients with invasive adenocarcinoma.

\section{Observation indicators}

The location and maximum diameter of the nodule and the distance between the nodule and the pleura were recorded in medical documents and the picture archiving and communication system prior to VATS. The localization effect was evaluated during VATS. The pathological result (benign or malignant) was documented following VATS. Patients were observed for complications, including pneumothorax, pulmonary hemorrhage, local pain, and cough.

The primary endpoints were the safety and the success rates of the 2 localization techniques. The secondary endpoints were operative time and feasibility. Successful localization should meet the following requirements: (I) the lesion is localized and resected quickly and accurately; (II) the distance between the marked and actual position of the lesion is $<10 \mathrm{~mm}$; (III) no malfunctions that lead to failure during localization; (IV) there is no displacement or dislocation of the needle; and (V) localization operations do not inhibit pathological observation. The definition of major and minor complications were in line with those of the Society of Interventional Radiology Classification System (10). The total complication rate was defined as the number of patients with complications in a group divided by the total number of patients in the same group.

\section{Statistical analysis}

SPSS version 13.0 (SPSS, Chicago, IL, USA) was used for the statistical analysis. The independent samples $t$-test was used for the comparison between the measurement data, and the $\chi^{2}$ test and Mann-Whitney $U$-test were used for the count data. Univariate analysis was performed for factors that were potentially related to post-localization complications, while logistic regression analysis was performed for factors that had a significant effect on the occurrence of complications. $\mathrm{P}<0.05$ indicated statistical significance. 
Table 1 Patients' clinical data

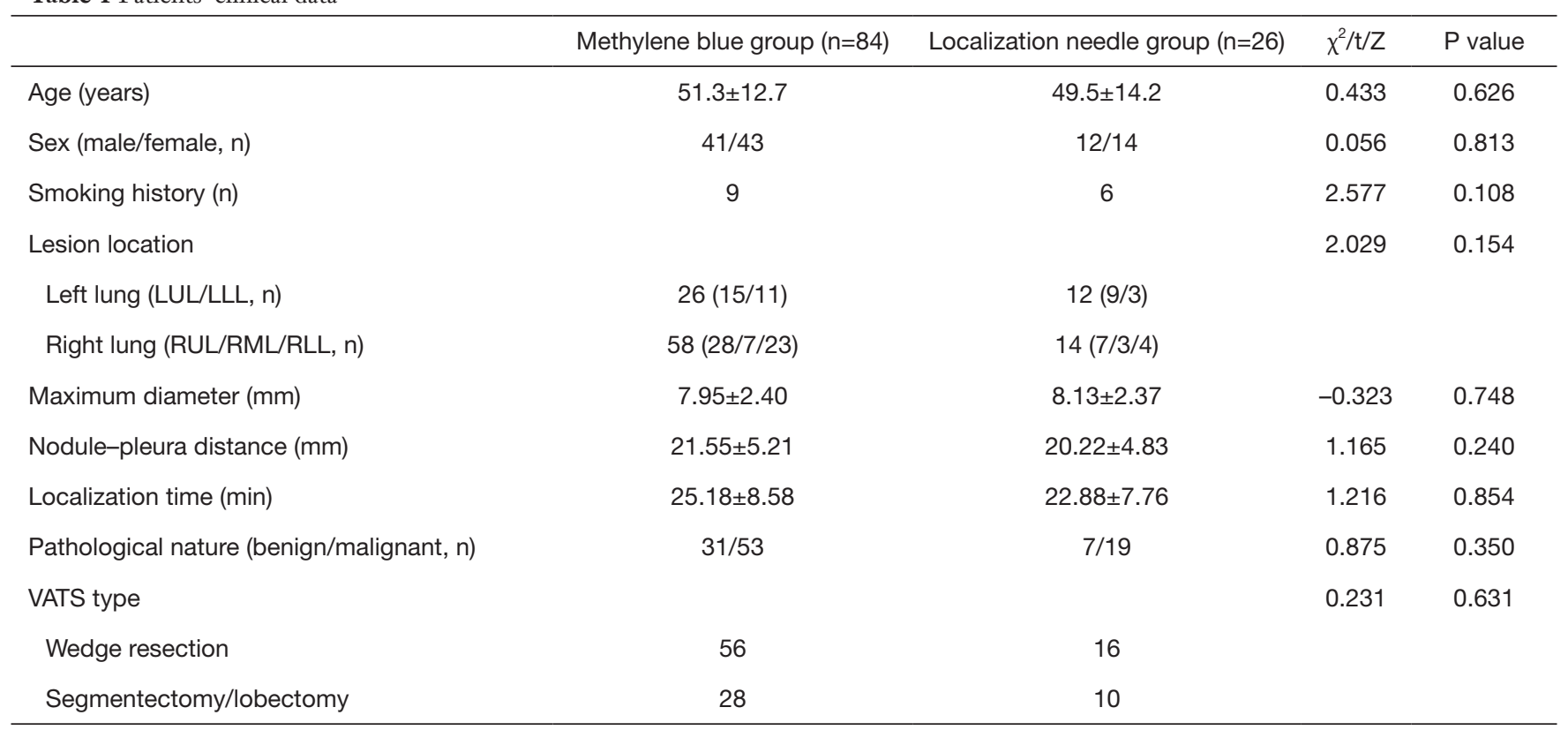

LLL, left lower lobe; LUL, left upper lobe; RLL, right lower lobe; RML, right middle lobe; RUL, right upper lobe; VATS, video-assisted thoracoscopic surgery.

\section{Results}

\section{Baseline statistics}

A total of 110 patients who underwent CT-guided localization and VATS in Shenzhen People's Hospital from January 2019 to December 2019 were enrolled in the present study. There were 53 males and 57 females with an average age of $50.9 \pm 13.0$ years (range, $24-77$ years). Of the cases, 83 were detected incidentally or during physical examination, 16 were because of recurrent cough, while the other 11 were because of chest pain and tightness. The 110 patients were categorized into 1 of 2 groups, depending on the localization technique employed: the MBSG group $(n=84)$ and the pulmonary nodule localization needle group $(n=26)$.

There were 41 males and 43 females, with an average age of $51.3 \pm 12.7$ years (range, $26-77$ years), in the MBSG group. Nine patients had a history of smoking; 56 patients had only 1 lesion, whereas the other 28 had at least 2 nodules. There were 12 males and 14 females, with an average age of $49.5 \pm 14.2$ years (range, $24-72$ years) in the pulmonary nodule localization needle group. Six patients had a history of smoking. The 26 cases consisted of 16 with a single lesion and 10 with multiple lesions $(\geq 2)$. Differences between the 2 groups were not statistically significant based on age, sex, history of smoking, lesion location, maximum diameter, nodule-pleura distance, pathological nature (benign/ malignant), and VATS resection type $(\mathrm{P}>0.05)$ (Table 1).

The success rate of pre-VATS localization was $100 \%$ in both groups. VATS for SPNs was performed successfully in both groups after localization.

No deaths or major complications occurred during localization. Complications occurred in 55 patients in the MBSG group and in 11 patients in the pulmonary nodule localization needle group; however, they did not require any special clinical treatment. Complications included pneumothorax, pulmonary hemorrhage, pain, and localization-induced cough. The rates of pneumothorax, pulmonary hemorrhage, and localization-induced cough were $38.1 \%, 25 \%$, and $7.14 \%$, respectively, in the MBSG group, and $26.92 \%, 19.23 \%$, and $0 \%$, respectively, in the pulmonary nodule localization needle group. Differences between the two groups were not statistically significant $(\mathrm{P}>0.05)$. Localization-induced cough occurred in 6 patients in the MBSG group, but no patients in the pulmonary nodule localization needle developed this complication. Although the statistical analysis revealed no difference in the rate of this complication between the two groups, on clinical observation, the incidence was obviously higher in MBSG group. The total complication rate and the incidence of pain were significantly lower in the pulmonary 
Table 2 Comparison of complications in the two groups

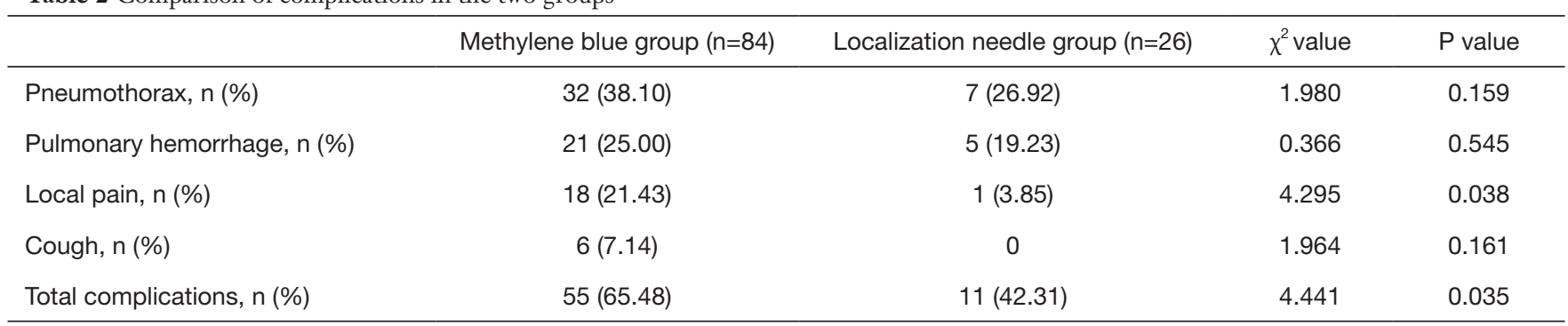

Table 3 Binary logistic regression analysis of factors associated with total complications

\begin{tabular}{lcccc}
\hline Factors & $\beta$ value & P value & Odds ratio & $95 \% \mathrm{Cl}$ \\
\hline Age & 0.014 & 0.374 & 1.014 & $0.983-1.046$ \\
Sex & -0.516 & 0.253 & 0.597 & $0.246-1.445$ \\
Smoking history & -0.487 & 0.445 & 0.614 & $0.176-2.145$ \\
Lesion location & -0.071 & 0.631 & 0.932 & $0.698-1.244$ \\
Maximum diameter & 0.012 & 0.889 & 1.012 & $0.851-1.205$ \\
Nodule-pleura distance & -0.012 & 0.765 & 0.988 & $0.912-1.070$ \\
Localization technique & 0.969 & 0.045 & 2.634 & $1.022-6.789$ \\
\hline
\end{tabular}

$\mathrm{Cl}$, confidence interval.

nodule localization needle group $\left(\chi^{2}=4.441\right.$ and 4.295 , respectively; $\mathrm{P}<0.05$ ) (Table 2).

The operative time was $25.18 \pm 8.58 \mathrm{~min}$ in the MBSG group and $22.88 \pm 7.76 \mathrm{~min}$ in the pulmonary nodule localization needle group $(\mathrm{P}>0.05)$. The interval between localization and VATS was 0.5 to $6 \mathrm{~h}$, during which there were no patients complained of chest pain. Dye diffusion was observed in 2 patients in the MBSG group (Figure 3); however, it had no impact on VATS or on the pathological analysis. Neither displacement nor dislocation was observed in the pulmonary nodule localization needle group. Logistic regression analysis showed that neither the total complication rate nor the rate of various complications was significantly associated with age, sex, smoking history, lesion location, maximum diameter, and nodule-pleura distance $(\mathrm{P}>0.05)$, and that the localization technique was an independent risk factor for total complications $(95 \%$ confidence interval: 1.022-6.789, $\mathrm{P}<0.05$ ) (Tables 3-7).

\section{Discussion}

As the clinical detection rate of SPNs increases, VATS is more widely used. However, it cannot accurately detect or localize SPNs that are $<1 \mathrm{~cm}$ in diameter or those that are located deep in the parenchyma ( $>5 \mathrm{~mm}$ from the pleura). In such cases, thoracotomy must be performed, which is a more invasive technique compared with VATS. It has been reported that the incidence of change from VATS to thoracotomy is up to $63 \%$ due to inaccurate localization or recognition of SPNs (11). Therefore, accurate pre-VATS localization is key to treatment success.

Multiple pre-VATS localization techniques have been used in the clinical setting. Of these, CT-guided percutaneous puncture technique is the most commonly used due to its simplicity, shorter operating time, and higher success rate. There are two ways to perform CT-guided percutaneous-puncture localization. These include injecting methylene blue or iodized oil, and implanting a hookwire or microcoil (12). However, both these techniques have limitations. Methylene blue injection is the first technique used for SPN localization. Its limitation is that it is prone to diffusion, resulting in localization difficulties (13). The surgeon's exposure to radiation is a major limitation of iodized oil injection. Microcoils may be left in the chest wall during localization of superficial pulmonary nodules. The incidence of pneumothorax, lung parenchyma hemorrhage, 
Table 4 Binary logistic regression analysis of factors associated with pneumothorax

\begin{tabular}{|c|c|c|c|c|}
\hline Factors & $\beta$ value & $P$ value & Odds ratio & $95 \% \mathrm{Cl}$ \\
\hline Sex & -0.370 & 0.413 & 0.691 & $0.285-1.676$ \\
\hline Smoking history & -0.076 & 0.912 & 0.926 & $0.239-3.594$ \\
\hline Lesion location & -0.127 & 0.389 & 0.881 & $0.660-1.176$ \\
\hline Nodule-pleura distance & 0.013 & 0.748 & 1.013 & $0.936-1.097$ \\
\hline Localization technique & 0.528 & 0.304 & 1.696 & $0.619-4.641$ \\
\hline
\end{tabular}

$\mathrm{Cl}$, confidence interval.

Table 5 Binary logistic regression analysis of factors associated with pulmonary hemorrhage

\begin{tabular}{lcccc}
\hline Factors & $\beta$ value & P value & Odds ratio & $95 \% \mathrm{Cl}$ \\
\hline Age & -0.001 & 0.966 & 0.999 & $0.965-1.035$ \\
Sex & -0.068 & 0.892 & 0.934 & $0.350-2.497$ \\
Smoking history & -1.361 & 0.221 & 0.257 & $0.029-2.266$ \\
Lesion location & -0.082 & 0.620 & 0.921 & 0.903 \\
Maximum diameter & -0.103 & 0.312 & 0.982 & $0.740-1.101$ \\
Nodule-pleura distance & -0.018 & 0.698 & $0.898-1.075$ \\
Localization technique & 0.176 & 0.765 & 1.192 & $0.377-3.767$ \\
\hline
\end{tabular}

$\mathrm{Cl}$, confidence interval.

Table 6 Binary logistic regression analysis of factors associated with local pain

\begin{tabular}{lcccc}
\hline Factors & $\beta$ value & P value & Odds ratio & 95\% Cl \\
\hline Age & 0.023 & 0.270 & 1.023 & $0.982-1.066$ \\
Sex & -0.600 & 0.295 & 0.549 & $0.179-1.688$ \\
Smoking history & -19.167 & 0.998 & - & 0.956 \\
Lesion location & -0.045 & 0.813 & 1.046 & $0.662-1,383$ \\
Maximum diameter & 0.045 & 0.702 & 0.968 & $0.832-1.315$ \\
Nodule-pleura distance & -0.032 & 0.536 & 0.423 & $0.875-1.072$ \\
Localization technique & 1.860 & 0.083 & & $0.784-52.623$ \\
\hline
\end{tabular}

$\mathrm{Cl}$, confidence interval.

and hookwire displacement is higher in patients undergoing hookwire localization (14).

CT-guided MBSG and CT-guided pulmonary nodule localization needle were introduced successively to our department. Surgical glue mainly contains N-butyl-2cyanoacrylate. It is widely used in clinical settings because of its good hemostatic performance. Under the influence of anions in the blood and tissue fluid around the wound, surgical glue generally begins to solidify within 3-5 s. After MBSG injection, SPNs and their surrounding tissues quickly solidify into hard nodules. This technique not only prevents methylene blue from diffusing and 
Table 7 Binary logistic regression analysis of factors associated with cough

\begin{tabular}{lcccc}
\hline Factors & $\beta$ value & P value & Odds ratio & 95\% Cl \\
\hline Age & 0.012 & 0.736 & 1.012 & $0.943-1.087$ \\
Sex & -1.475 & 0.228 & 0.229 & $0.021-2.512$ \\
Smoking history & 1.240 & 0.413 & 3.456 & $0.178-67.253$ \\
Lesion location & 0.019 & 0.958 & 1.019 & $0.505-2.055$ \\
Maximum diameter & 0.422 & 0.066 & 1.525 & $0.972-2.392$ \\
Nodule-pleura distance & -0.108 & 0.264 & 0.897 & $0.742-1.085$ \\
Localization technique & 18.538 & 0.998 & $1 \mathrm{E}+008$ & - \\
\hline
\end{tabular}

$\mathrm{Cl}$, confidence interval.

facilitates localization through palpation, but also reduces the risk of puncture-induced bleeding and pneumothorax. However, there are 2 major problems associated with this technique; cough and chest pain may occur, and sampling for pathological examination may be inhibited due to methylene blue diffusion.

In 2019, Fan et al. developed a novel disposable pulmonary nodule localization needle (Ningbo Senscure Biotechnology, Ningbo, China) based on a hookwire (9). The use of this novel needle reduces the incidence of hookwire-induced complications, such as pain, and hookwire displacement and dislocation. The device consists of five parts; the coaxial needle, pusher, anchor claw, suture, and protection tube. The cannula of the needle is marked with a scale to determine the distance between SPNs and the pleura according to CT images. The anchor claw is made of nickel titanium, with four blunt-ended fishhookshaped hooks that form a cross. The suture connected to the anchor claw is made of polyethylene terephthalate (absorbable), and consists of three segments. The three parts are colored differently, making it easy for surgeons to calculate how deep the needle is inserted according to the part of the suture left outside the body (Figure 2). This novel device can potentially reduce the incidence of hookwire displacement and dislocation. Because the anchor claw can withstand considerable traction, it can be used to pull lung tissues and resect nodules, maximizing the preservation of lung tissues. The scale on the cannula helps place the device effectively and accurately. Patients can well tolerate the absorbable suture, experiencing no infection, dyspnea, cough, or chest pain after localization.

In the present study, SPNs were successfully localized in both groups (100\%). The two groups also had similar operative times $(t=1.216, P>0.05)$, indicating that the success rates of the two techniques are similar in the clinical setting.

The MBSG technique has been proven to be safe (15). Common complications of this technique include pneumothorax and bleeding (16). The findings of the present study suggest that the 2 groups are similar in the rates of pneumothorax $\left(\chi^{2}=1.980, \mathrm{P}=0.159\right)$ and bleeding $\left(\chi^{2}=0.366\right.$, $\mathrm{P}=0.545)$. These 2 complications only present mild clinical symptoms and do not require treatment.

In the present study, localization-induced cough only occurred in MBSG group (6/84, 7.14\%). Two potential causes may be the smell of the two fluids and their entry into small airways along the puncture route. Although no patient experienced cough in the pulmonary nodule localization needle group, the statistical analysis revealed no significant difference between the two groups in the rate of this complication $\left(\chi^{2}=1.964, \mathrm{P}=0.161\right)$. Although the incidence of localization-induced cough was low, the rate in the novel localization needle was lower.

Post-localization pain is also worthy of attention. In the present study, the incidence of pain was lower in the pulmonary nodule localization needle group than the MBSG group $\left(3.85 \%\right.$ vs. $\left.21.43 \%, \chi^{2}=4.295 ; \mathrm{P}=0.038\right)$. Most of the patients who experienced pain underwent MBSG localization is mainly concentrated in the initial stage of using this method, the authors realized that the cause of pain might be reflux of surgical glue and methylene blue along the puncture route into the visceral pleura. Attention was then paid to controlling the overall usage of the MBSG, reducing the injection speed, and avoiding injection into the visceral pleura. The rate of pain then decreased. Only 1 patient in the pulmonary nodule localization needle group experienced post-localization pain. Therefore, pulmonary nodule localization needle can reduce the incidence of pain significantly.

Previously published studies have reported that the 


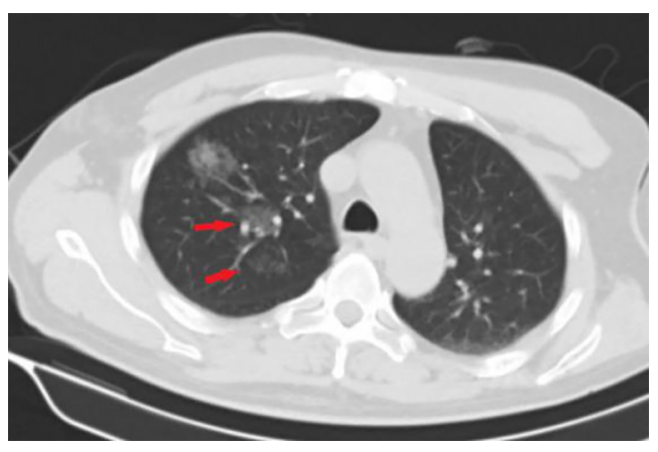

Figure 3 Computed tomography (CT) image of small pulmonary nodules in the right upper lung lobe of a 48-year-old male. After injection of the suspension of surgical glue and methylene blue, diffusion of methylene blue into lung tissues is observed (red arrow).

diffusion of methylene blue in the lung parenchyma makes it difficult to determine the location of SPNs, and their relationship with surrounding structures. They recommend that VATS should be performed within $3 \mathrm{~h}$ following methylene blue injection $(17,18)$. In the present study, methylene blue was used together with surgical glue to avoid diffusion; however, diffusion was still observed in 2 patients during VATS (Figure 3). Although surgery and pathological diagnoses were not affected, it is still recommended that the 2 fluids are completely mixed and that VATS is performed without delay. In the pulmonary nodule localization needle group, SPNs were localized accurately, and neither displacement nor dislocation was observed.

Post-localization complications depend on various factors, such as the operator's experience, nodule size, nodule location, nodule-pleura distance, and patients' compliance $(19,20)$. The findings of the present study indicated that difference in the total complication rate between the two groups was statistically significant $\left(\chi^{2}=4.441, \mathrm{P}=0.035\right)$. Further statistical analyses showed that neither the total complication rate nor the rate of various complications was significantly associated with age, sex, history of smoking, lesion location, maximum diameter, and nodule-pleura distance $(\mathrm{P}>0.05)$. Findings of the multivariate analysis suggest that the localization technique is an independent risk factor for total complications (odds ratio: 2.634, 95\% confidence interval: $1.022-6.789, \mathrm{P}=0.045)$. The total complication rate was lower in the pulmonary nodule localization needle group than the MBSG group.

The present study has some limitations. First, this was not a randomized study, and there was a significant difference in the number of patients between the two groups. Second, the sample size was small. Finally, all the patients were from the same hospital. Multicenter randomized controlled studies with larger sample sizes are warranted to confirm the results of the present study.

\section{Conclusions}

Both the pulmonary nodule localization needle and the MBSG techniques can help localize SPNs effectively and quickly. The novel needle technique is associated with a lower incidence of complications; therefore, is safe and effective for SPN treatment.

\section{Acknowledgments}

Funding: The present study received honoraria from the Subject Ability Improvement Project of Shenzhen Health and Fitness Commission, The Research and Development of Augmented Reality Navigation System for Precise Ablation of Liver Cancer (No. SZXJ2017066), and Shenzhen Key Medical Discipline Construction Fund (No. SZXK018).

\section{Footnote}

Reporting Checklist: The authors have completed the TREND reporting checklist. Available at http://dx.doi. org/10.21037/jtd-20-3147

Data Sharing Statement: Available at http://dx.doi. org/10.21037/jtd-20-3147

Conflicts of Interest: All authors have completed the ICMJE uniform disclosure form (available at http://dx.doi. org/10.21037/jtd-20-3147). The authors have no conflicts of interest to declare.

Ethical Statement: The authors are accountable for all aspects of the work in ensuring that questions related to the accuracy or integrity of any part of the work are appropriately investigated and resolved. This present study was reviewed and approved by the Ethics Committee of Shenzhen People's Hospital, China (No. LL-KY-2020325). Signed informed consent was provided by patients and/or immediate family members preoperatively. All procedures performed in this study involving human participants were in accordance with the Declaration of Helsinki (as revised in 2013).

Open Access Statement: This is an Open Access article distributed in accordance with the Creative Commons 
Attribution-NonCommercial-NoDerivs 4.0 International License (CC BY-NC-ND 4.0), which permits the noncommercial replication and distribution of the article with the strict proviso that no changes or edits are made and the original work is properly cited (including links to both the formal publication through the relevant DOI and the license). See: https://creativecommons.org/licenses/by-nc-nd/4.0/.

\section{References}

1. Naidich DP, Bankier AA, MacMahon H, et al. Recommendations for the management of subsolid pulmonary nodules detected at CT: a statement from the Fleischner Society. Radiology 2013;266:304-17.

2. NCCN Clinical Practice Guidelines in Oncology. Lung Cancer Screening. v1, 2020. Available online: www.nccn. org/professionals/physician_gls/f_guidelines.asp

3. Kothary N, Lock L, Sze DY, et al. Computed tomographyguided percutaneous needle biopsy of pulmonary nodules: impact of nodule size on diagnostic accuracy. Clin Lung Cancer 2009;10:360-3.

4. Khandhar SJ, Bowling MR, Flandes J, et al. Electromagnetic navigation bronchoscopy to access lung lesions in 1,000 subjects: first results of the prospective, multicenter NAVIGATE study. BMC Pulm Med 2017;17:59.

5. Mack MJ, Hazelrigg SR, Landreneau RJ, et al. Thoracoscopy for the diagnosis of the indeterminate solitary pulmonary nodule. Ann Thorac Surg 1993;56:82530; discussion 30-2.

6. Mei J, Guo C, Xia L, et al. Long-term survival outcomes of video-assisted thoracic surgery lobectomy for stage I-II non-small cell lung cancer are more favorable than thoracotomy: a propensity score-matched analysis from a high-volume center in China. Transl Lung Cancer Res 2019;8:155-66.

7. Tsai TM, Chiang XH, Liao HC, et al. Computed tomography-guided dye localization for deeply situated pulmonary nodules in thoracoscopic surgery. Ann Transl Med 2019;7:31.

8. Ichinose J, Kohno T, Fujimori S, et al. Efficacy and complications of computed tomography-guided hook wire localization. Ann Thorac Surg 2013;96:1203-8.

9. Fan L, Yang H, Yu L, et al. Multicenter, prospective, observational study of a novel technique for preoperative pulmonary nodule localization. J Thorac Cardiovasc Surg 2020;160:532-39.e2.

10. Omary RA, Bettmann MA, Cardella JF, et al. Quality improvement guidelines for the reporting and archiving of interventional radiology procedures. J Vasc Interv Radiol 2003;14:S293-5.

11. Suzuki K, Nagai K, Yoshida J, et al. Video-assisted thoracoscopic surgery for small indeterminate pulmonary nodules: indications for preoperative marking. Chest 1999;115:563-8.

12. McDermott S, Fintelmann FJ, Bierhals AJ, et al. Imageguided Preoperative Localization of Pulmonary Nodules for Video-assisted and Robotically Assisted Surgery. Radiographics 2019;39:1264-79.

13. Fumimoto S, Sato K, Koyama M, et al. Combined lipiodol marking and video-assisted thoracoscopic surgery in a hybrid operating room. J Thorac Dis 2018;10:2940-7.

14. Lempel JK, Raymond DP, Ahmad U, et al. Video-Assisted Thoracic Surgery Resection without Intraoperative Fluoroscopy after CT-Guided Microcoil Localization of Peripheral Pulmonary Nodules. J Vasc Interv Radiol 2018;29:1423-28.

15. Lin FC, Tsai SC, Tu HT, et al. Computed tomographyguided localization with laser angle guide for thoracic procedures. J Thorac Dis 2018;10:3824-8.

16. Lin MW, Chen JS. Image-guided techniques for localizing pulmonary nodules in thoracoscopic surgery. J Thorac Dis 2016;8:S749-55.

17. Lee NK, Park CM, Kang CH, et al. CT-guided percutaneous transthoracic localization of pulmonary nodules prior to video-assisted thoracoscopic surgery using barium suspension. Korean J Radiol 2012;13:694-701.

18. Keating J, Singhal S. Novel Methods of Intraoperative Localization and Margin Assessment of Pulmonary Nodules. Semin Thorac Cardiovasc Surg 2016;28:127-36.

19. Zaman M, Bilal H, Woo CY, et al. In patients undergoing video-assisted thoracoscopic surgery excision, what is the best way to locate a subcentimetre solitary pulmonary nodule in order to achieve successful excision? Interact Cardiovasc Thorac Surg 2012;15:266-72.

20. Hsu HH, Shen CH, Tsai WC, et al. Localization of nonpalpable pulmonary nodules using CT-guided needle puncture. World J Surg Oncol 2015;13:248.

(English Language Editor: R. Scott)

Cite this article as: Kong J, Guo J, Zhang H, Li Y, Wang G, Zhang Y. CT-guided localization techniques of small pulmonary nodules: a prospective non-randomized controlled study on pulmonary nodule localization needle and methylene blue staining with surgical glue. J Thorac Dis 2020;12(11):68266835. doi: $10.21037 /$ jtd-20-3147 